\title{
BMJ Open Cohort profile of Acutelines: a large data/biobank of acute and emergency medicine
}

\author{
Ewoud ter Avest (D) , ${ }^{1}$ Barbara C van Munster, ${ }^{2}$ Raymond J van Wijk (D) , \\ Sanne Tent, ${ }^{2}$ Sanne Ter Horst, ${ }^{2}$ Ting Ting Hu, ${ }^{2}$ Lisanne E van Heijst, ${ }^{2}$ \\ Felien S van der Veer, ${ }^{2}$ Fleur E van Beuningen, ${ }^{2}$ Jan Cornelis ter Maaten (D) , ${ }^{3}$ \\ Hjalmar R Bouma ${ }^{2,4}$
}

\begin{abstract}
To cite: ter Avest $\mathrm{E}$, van
Munster BC, van Wijk RJ, et al. Cohort profile of Acutelines: a large data/biobank of acute and emergency medicine. BMJ Open 2021;11:e047349. doi:10.1136/ bmjopen-2020-047349

- Prepublication history and additional online supplemental material for this paper are available online. To view these files, please visit the journal online (http://dx.doi.org/10. 1136/bmjopen-2020-047349)
\end{abstract}

Received 29 November 2020 Accepted 01 July 2021

Check for updates

(C) Author(s) (or their employer(s)) 2021. Re-use permitted under CC BY-NC. No commercial re-use. See rights and permissions. Published by BMJ.

${ }^{1}$ Emergency medicine, University Medical Centre Groningen, Groningen, The Netherlands ${ }^{2}$ Internal Medicine, University Medical Centre Groningen, Groningen, The Netherlands ${ }^{3}$ Emergency Department \& Department of internal medicine, University Medical Centre Groningen, Groningen, The Netherlands

${ }^{4}$ Clinical Pharmacy and Pharmacology, University Medical Centre Groningen, Groningen, The Netherlands

Correspondence to

Dr Ewoud ter Avest;

e.ter.avest@umcg.nl

\section{ABSTRACT}

Purpose Research in acute care faces many challenges, including enrolment challenges, legal limitations in data sharing, limited funding and lack of singular ownership of the domain of acute care. To overcome these challenges, the Center of Acute Care of the University Medical Center Groningen in the Netherlands, has established a de novo data, image and biobank named 'Acutelines'.

Participants Clinical data, imaging data and biomaterials (ie, blood, urine, faeces, hair) are collected from patients presenting to the emergency department (ED) with a broad range of acute disease presentations. A deferred consent procedure (by proxy) is in place to allow collecting data and biomaterials prior to obtaining written consent. The digital infrastructure used ensures automated capturing of all bed-side monitoring data (ie, vital parameters, electrophysiological waveforms) and securely importing data from other sources, such as the electronic health records of the hospital, ambulance and general practitioner, municipal registration and pharmacy. Data are collected from all included participants during the first 72 hours of their hospitalisation, while follow-up data are collected at 3 months, 1 year, 2 years and 5 years after their ED visit.

Findings to date Enrolment of the first participant occurred on 1 September 2020. During the first month, 653 participants were screened for eligibility, of which 180 were approached as potential participants. In total, $151(84 \%)$ provided consent for participation of which 89 participants fulfilled criteria for collection of biomaterials. Future plans The main aim of Acutelines is to facilitate research in acute medicine by providing the framework for novel studies and issuing data, images and biomaterials for future research. The protocol will be extended by connecting with central registries to obtain long-term follow-up data, for which we already request permission from the participant.

Trial registration number NCT04615065.

\section{INTRODUCTION}

Research in acute care is important to prevent diseases, and to establish best practices for treatments of many acute conditions. Over the past 50 years, medical knowledge has grown exponentially, and specialties as
Strengths and limitations of this study

- Presence of a dedicated research team to screen and include patients in the emergency department and perform data entry, quality control and quality assurance.

- Deferred consent procedure, when applicable by proxy, to allow the collection of data and biomaterials prior to obtaining consent.

- Digital infrastructure to automatically capture all bed-side monitor data (ie, electrophysiological waveforms, vital parameters) from every patient in the emergency department.

- Software to securely connect with other sources, such as the electronic health records of the hospital, ambulance and general practitioner, municipal registration, health insurance companies and pharmacy.

- A potential limitation is the relative inefficiency as patients are included based on broad inclusion criteria (ie, transport, urgency, complaints), while data and biomaterials will mostly be used for subsequent studies based on specific discharge diagnosis.

emergency Mmedicine and acute internal medicine have developed rapidly. ${ }^{1}$ Despite this, knowledge gaps still exist. Among these are logistics of care (optimal patient disposition, triage, and prevention of crowding), the development of tools for early recognition of acutely sick patients (including risk prediction models, biomarkers and/or artificial intelligence (AI)), and the development of more patient centred and personalised care for specific subgroups presenting in the emergency department (ED), such as the frail elderly population, patients with psychiatric illnesses and patients with (early) sepsis. ${ }^{2-4}$

However, conducting research in acute care may prove difficult for various reasons. First, enrolment challenges often limit the number of participants eligible to participate in clinical studies. ${ }^{56}$ As many acute conditions are time sensitive and expedited diagnostic 
evaluation and/or initiation of treatment is warranted, this is normally prioritised over study enrolment. Further, obtaining consent from acutely ill patients to participate in clinical studies can be challenging or even impossible, especially when a proxy or legal representative is not available to emergency medical services (EMS) or ED personnel to discuss study aims- and risks within the enrolment window. ${ }^{78}$ Finally, availability of staff for enrolment of participants can be an issue with increasing pressure on healthcare systems and the resultant crowding of ED's in many countries. ${ }^{9}$

Second, in order to gain more insight into the natural course of acute diseases, and to be able to investigate which patients will deteriorate rapidly during the course of their disease, it is important to collect data from the start of their disease onwards until they are fully recovered or died. However, this is often difficult to achieve due to legal limitations in data sharing. Data transfer for clinical research between various organisation involved in the care for a particular patient and the secondary research use of data and or biomaterials can only occur with explicit permission of the patient. ${ }^{10}$ As with consent, timely permission is often not possible to obtain. Further, no single specialism is the 'owner' of acute care. Although the organisation of healthcare systems may vary from one country to the other, acute care is multidisciplinary by nature. Research interests and priorities may vary between specialties involved, which may complicate conduct of clinical studies. Finally, it can be a challenge to obtain funding for acute care research. Although researchers may apply for government sponsored grants of organisations as the National Institute of Health or the Dutch research Council (NWO), the number of grants funded by these institutions related to acute care are low, ${ }^{11}$ and additional funding is often required.

In an attempt to overcome some of these aforementioned challenges, the Center of Acute Care of the University hospital Groningen in the Netherlands, has established a de novo data, image and biobank named 'Acutelines'. Acutelines is unique, as it is not aimed at one specific disease or condition as other critical care biobanks, ${ }^{12}{ }^{13}$ but instead collects data, imaging and biomaterials from as many patients presenting with acute conditions as possible, aiming to explore the association between pre-existing health, acute illness and (long-term) outcome. (figure 1). The objective of this manuscript is to describe the process by which we established Acutelines, and how we integrated operations into ED clinical workflow allowing Acutelines to operate 24 hours a day, 7 days a week $(24 / 7)$.

\section{COHORT DESCRIPTION Study design}

Acutelines is a prospective data, image and biobank including patients with a broad spectrum of acute conditions. Its aim is to facilitate interdisciplinary research on the aetiology and development of acute diseases with the aid of systematically collected biomaterials and medical data over various time points, both during the course

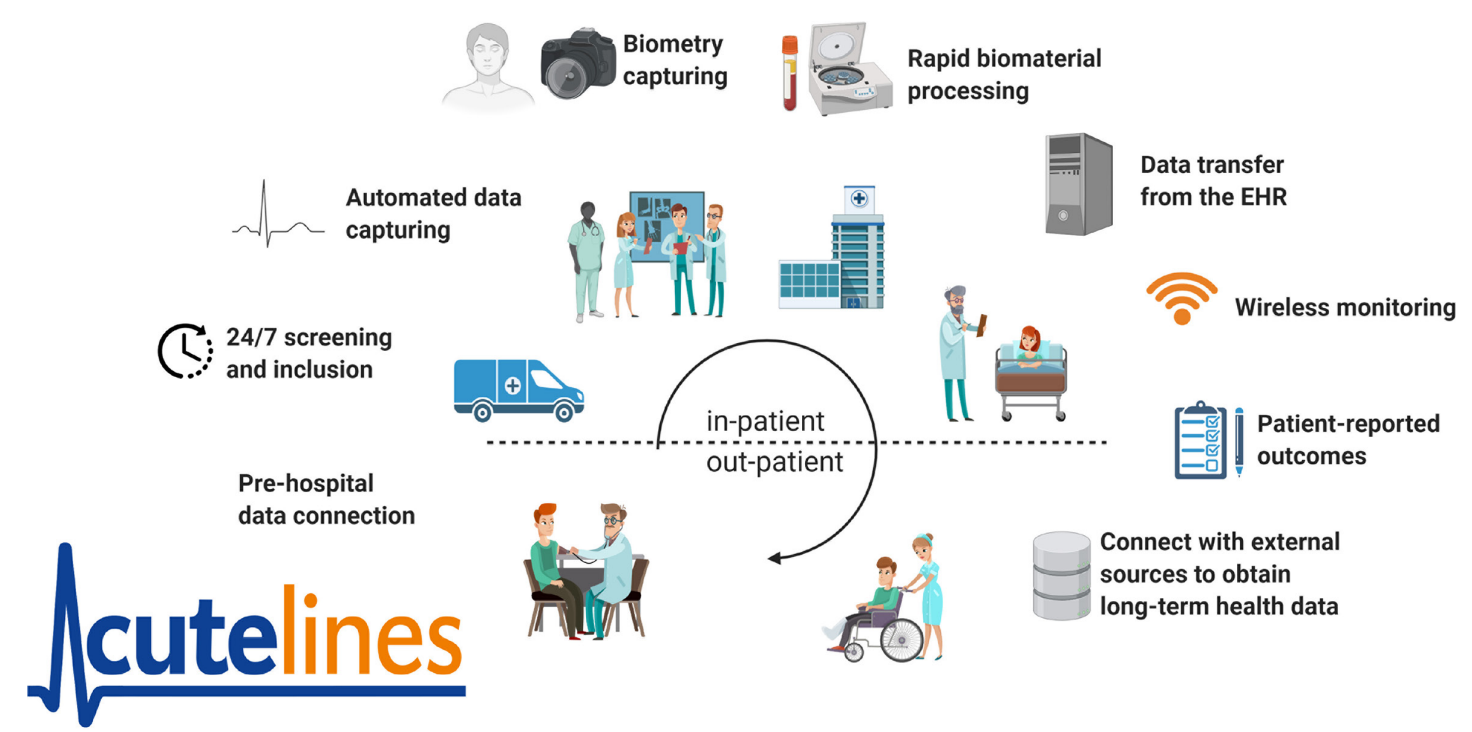

Figure 1 Schematic overview of the Acutelines biobank. By collecting data from prehospital up to long after hospital discharge, Acutelines follows the complete acute patient journey. Specially trained research assistants screen potential participants in the emergency department (ED) (24/7). Waveform data and vital parameters from bed-side monitors are captured automatically, and biomaterials (ie, blood, urine, faeces) will be collected while awaiting deferred consent (by proxy). ED facilities allow rapid processing and storage of biomaterials $\left(-80^{\circ} \mathrm{C}\right)$. Wearable devices are used to continue capturing waveforms and vital parameters during the first 72 hours of hospital admission. Connections with the electronic health record and external databases (eg, GP, pharmacy, health insurance companies) allow to collect relevant clinical data when applicable for specific research questions, such as medication use and comorbidity up to 5 years after presentation. Digital survey-based patientreported outcomes will be collected on fixed intervals and survival will be monitored indefinitely using the municipal registration. GP, general practitioner. 
Table 1 WHO trial registration dataset

Primary registry and trial identifying number

Date of registration in primary registry

Secondary identifying numbers

Sources of monetary of material support

Primary sponsor

Secondary sponsor

Contact for public queries

Contact for research queries

Public title

Scientific title

Country of recruitment

Health conditions

Interventions

Key inclusion-exclusion criteria

(biobank protocol version 5, 09-2020)
ClinicalTrials.gov: NCT04615065

18 October 2020

University Medical Centre Groningen Research Registry Number 201900635

University Medical Centre Groningen, University of Groningen, The Netherlands University Medical Centre Groningen, University of Groningen, The Netherlands None

Acutelines steering group (acutelines@umcg.nl)

Acutelines steering group (acutelines@umcg.nl)

Acutelines

Acutelines de novo data/biobank

Netherlands

Acute conditions

None

Included are ED patients with the highest and second highest urgency triage categories of the Manchester triage system, and patients with the third highest category when arriving by (Helicopter) emergency medical service. In addition, patients with several specific conditions regardless their triage category are included: Sepsis, shock, syncope, anaphylaxis, acute renal failure, intoxications, COPD exacerbations, deep venous thrombosis or pulmonary embolism, gastrointestinal bleeding and patients who are bleeding (any source) while using warfarin or DOAC.

Study Type

Observational, biobank

Date of first enrolment

1 September 2020

Sample size

Recruitment status

Anticipated at 3500 per year

Recruiting
Ethics approval

Status: approved

Date of approval: 8 April 2020

Name and contact details of ethics committees:

Institutional Review Board and Central Review Board

University Medical Centre Groningen

Phone:+31503613564

Email: nwmoloket@umcg.nl

Address: PO Box 30001

9700 RB

Groningen

The Netherlands

Completion Date

Not applicable (no end date defined)

Summary statement

IPD sharing statement
No results yet

Individual participant data (IPD) may be available to other researchers if needed for their specific research purposes, which among others must be in line with the study protocol, the informed consent form and the general data protection regulations. Each request for reuse of data will be reviewed by Acutelines' steering group, manager and local review board, prior to establishing a material and data transfer agreement. No IPD will be shared if not required to answer research question.

ED, emergency department.

of the patient's disease and after recovery. Acutelines is initiated by the departments of Emergency Medicine and Internal Medicine of the University Medical Center Groningen (UMCG), the Netherlands, and registered in ClinicalTrials.gov (see table 1 for WHO trial registration dataset) and in the Groningen data catalogue. The latest biobank protocol and regulations are accessible via http://acutelinesumcgnl. The biobank is governed by a three-person scientific committee (HRB, JCtM and EtA) with oversight of a trustee (BCvM) and supported by two data managers (RJvW and ST) and a team of research 
assistants, led by team captains (STH, TTH, LEvH FEvB and FSvdV).

\section{Setting}

All patients admitted to the ED of the UMCG, a large tertiary care centre with approximately 26000 ED visits per year, for specialties participating in Acutelines are screened for eligibility. Participating specialties during the (current) initiation phase of the biobank are: emergency medicine, internal medicine (including subspecialisations as allergology, acute medicine, oncology, haematology, infectiology, nephrology, vascular medicine, geriatric medicine), pulmonology, gastroenterology and rheumatology. The scientific board of Acutelines aims to allow participation of other specialties as well in the near future after the initiation phase. The ultimate goal of Acutelines is to generate a biobank for acute diseases with a high scientific merit, that is, logistically efficient and financially sustainable, and can become a resource to both academic and industry partners. The sample size of the biobank is directly related to available funding, which allows us to include approximately 3500 participants/year in the initiation phase. This number is likely to expand once more specialties will participate, and/or when external parties will apply for the use of data and samples from the bank. The first participant was enrolled on 1 September 2020. Acutelines does not have a fixed end date or sample size.

\section{Eligibility criteria}

To be eligible for inclusion, according to the latest version of the Acutelines protocol participants have to meet at least one of the following criteria (figure 2):

- Patients with the highest and second highest urgency triage categories (red or orange) of the Emergency Severity Index (ESI). ${ }^{14}$
- Patients with the third highest category urgency triage category (yellow) of the ESI when arriving by (Helicopter) EMS.

- Patients with several specific conditions regardless their triage category are included: sepsis, shock, syncope, anaphylaxis, acute renal failure, electrolyte disturbances intoxications, Chronic Obstructive Pulmonary Disease (COPD) and asthma exacerbations, (suspicion of) deep venous thrombosis or pulmonary embolism, gastrointestinal bleeding and patients who are bleeding (any source) while using vitamin-K antagonists or Direct Oral Anticoagulants (DOAC).

While data and imaging will be collected from all included participants, biomaterials will only be collected from participants fulfilling the first criterion and from participants with shock or a suspicion of sepsis (irrespective of their triage category, figure 1). Criteria to define sepsis and shock are intentionally left broad in order to recruit participants who might present with aspecific complaints and in addition include cases to serve as controls in future studies as well. Sepsis is defined based on either the physician's gestalt, ${ }^{15}$ or when sepsis- 2 or sepsis-3 criteria are met. ${ }^{16}$ Shock is defined as hypotension (systolic blood pressure (BP) $<90 \mathrm{~mm} \mathrm{Hg}$ or a decrease of $>40 \mathrm{~mm} \mathrm{Hg}$ compared with pre-existent) in combination with tachycardia (HR $>100$ beats/min). Inclusion criteria will be adapted and broadened with each new specialty participating in the biobank.

\section{Participant recruitment}

In order to allow biomaterial collection when applicable on first contact, primary screening of patients for eligibility on arrival in the ED is performed $24 / 7$ by the ED-(triage)nurse together with a trained dedicated research team (ie, research assistants), and it is the ambition to recruit patients $24 / 7$ staffing allowed. After

Adult patients visiting emergency room for: internal medicine (and subspecialities), gastro-enterology, pulmonology, rheumatology, emergency medicine (non-trauma)

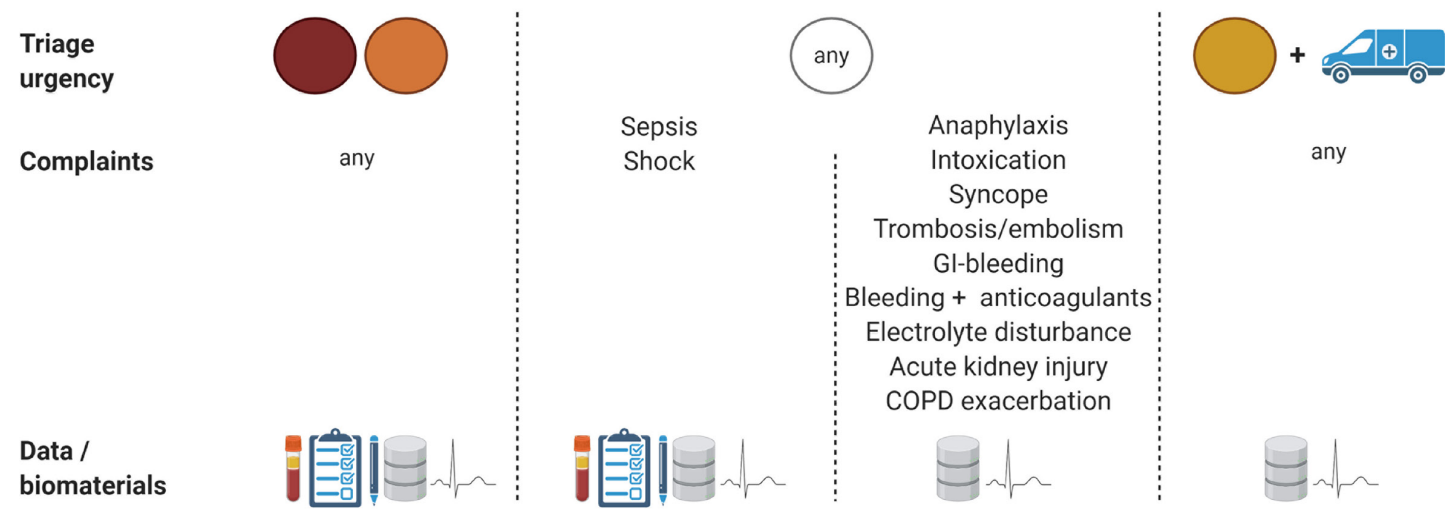

Figure 2 Inclusion criteria of patients for Acutelines in the initiation phase. Data (demographic and medical data, waveforms) will be collected from all patients, but surveys and biomaterials will only be collected from patients with the highest and second highest urgency triage categories (red or orange) of the Emergency Severity Index or patients with a suspicion of sepsis or shock. Blood tube: biomaterials, pen/paper: survey, database: health data (ie, from EHR and central registries), ECG: waveform data with vital parameters. EHR, electronic health record; GI, gastrointestina; COPD, Chronic Obstructive Pulmonary Disease 
identification of potentially eligible participants, the ED nurse will briefly inform participants about Acutelines. If the potential participant or his/her proxy does not refuse potential participation at that stage, blood specimens for Acutelines are collected during venipuncture for regular care. Subsequently, the research assistant informs participants and their relatives, obtains consent (by proxy), and collects and processes data and biomaterials in the ED.

\section{Data collection}

In total, four team captains perform quality control by verifying all entered data according to a data verification protocol, while data managers periodically perform quality assurance of entered and imported data. Most data are captured automatically either from the hospital electronic health record (EHR; EPIC) or directly from bed-side monitors (electrophysiological waveforms and vital parameters). Surveys are used to obtain information regarding health status, frailty (if age $\geq 70$ ), mood and depression, cognitive function (if age $\geq 70$ years) and physical activities. ${ }^{17-23}$ Surveys are filled in by participants (whenever possible) or their relatives using a tablet device at the time of presentation in the ED, their smartphone (after scanning a QR-code) or using pen/paper. Digital responses are entered directly into the research database, while paper responses will be entered by the research assistant and verified by the team captain. In order to obtain information about comorbidity and medication use at the moment of presentation, data are imported from other healthcare providers and from central registries when corresponding consent is given.

Study data are collected and managed using Research Electronic Data Capture (REDCap) electronic data capture tools hosted at UMCG, either by direct entry by research assistant, participant or next of kin; or by importing data from EPIC. ${ }^{24}{ }^{25}$ REDCap is a secure, webbased software platform designed to support data capture for research studies, providing an intuitive interface for validated data capture, audit trails for tracking data manipulation and export procedures, automated export procedures for seamless data downloads to common statistical packages and procedures for data integration and interoperability with external sources. Traffic between REDCap and the web browser is encrypted, and data are directly stored on a database server located in the UMCG and protected by a firewall. These systems are compliant with Good Clinical practice and are ISO 27001 certified. All participants are registered under a study number, which is used during both data collection and data processing. Table 2 and online supplemental figures 1 and 2 provide an overview of data collection at various time points. Acutelines' complete protocol and the actual, full data dictionary is available via https:// acutelines.umcg.nl, while a summarised version of the data dictionary is shown in online supplemental document 1.

\section{Vital parameters and cardiorespiratory waveforms}

Vital parameters (respiration rat, oxygen saturation, BP, heart rate (HR), Glasgow Coma Scale and temperature are collected from prehospital data provided at handover, and the first set of observations at triage in the ED is registered. In addition, high frequency (100$500 \mathrm{~Hz}$ ) cardiorespiratory waveforms (ECG, arterial BP, photoplethysmogram, transthoracic impedance and airway flow and pressure) are recorded continuously for all participants from the moment of arrival-until discharge from the ED. Availability of waveforms is dependent on necessity for standard care. Waveform data are transferred from the monitoring equipment (Philip IntelliVue MX550, Koninklijke Philips N.V., Eindhoven, the Netherlands) and ventilators (Dräger Primus, Dräger, Lubeck, Germany), to a waveform platform using a hub (Capsule Neuron, CapsuleTech) with dedicated drivers (Enovation) and automatically uploaded in the bank database. Waveform data are highly valuable for development of outcome prediction models and to monitor effects of ED interventions in future studies.

\section{Biomaterials}

During the initiation phase, we will collect four types of biomaterials from participants: blood, urine, faeces and hair. Once inclusion criteria are met, the following blood samples for Acutelines are drawn combined with regular blood sampling as part of standard clinical care (to streamline research efforts with clinical workflow and to avoid extra vena punctures): Plasma (citrate, EDTA, Li-heparin), Buffy coat (collected from EDTA), Serum (clot tube) and Whole blood (PAXgene RNA). In addition, the participant is asked to donate urine and faeces (at the time of or within 24 hours of ED visit) and hair. The collected materials are processed in the ED and stored by trained research assistants, in accordance with Acutelines standard operating procedures and applicable hospital regulations: samples are logged into the REDCap database where the link between participant identity and sample barcodes is stored. Pseudonymised samples are then aliquoted into $2.0 \mathrm{~mL}$ QR-coded screwcap microtubes (Sarstedt, Nümbrecht, Germany) and subsequently stored in the UMCG Central Freezer Facility at $-80^{\circ} \mathrm{C}$. The location of samples will be registered in the biobank information management system, which is only accessible to researchers associated with the project.

\section{Imaging data}

Indication, type of imaging performed and conclusion arising from imaging studies performed during the ED visit (including point-of-care ultrasound carried out for clinical and research purposes, X-rays and CT scans) are read from the reports by research assistants and entered in the databank. Related images are stored on the hospital Picture Archiving and Communication System system, and linked to the Acutelines database via a unique accession number. Additionally, to be able to unravel the factors that contribute to the phycisians' 


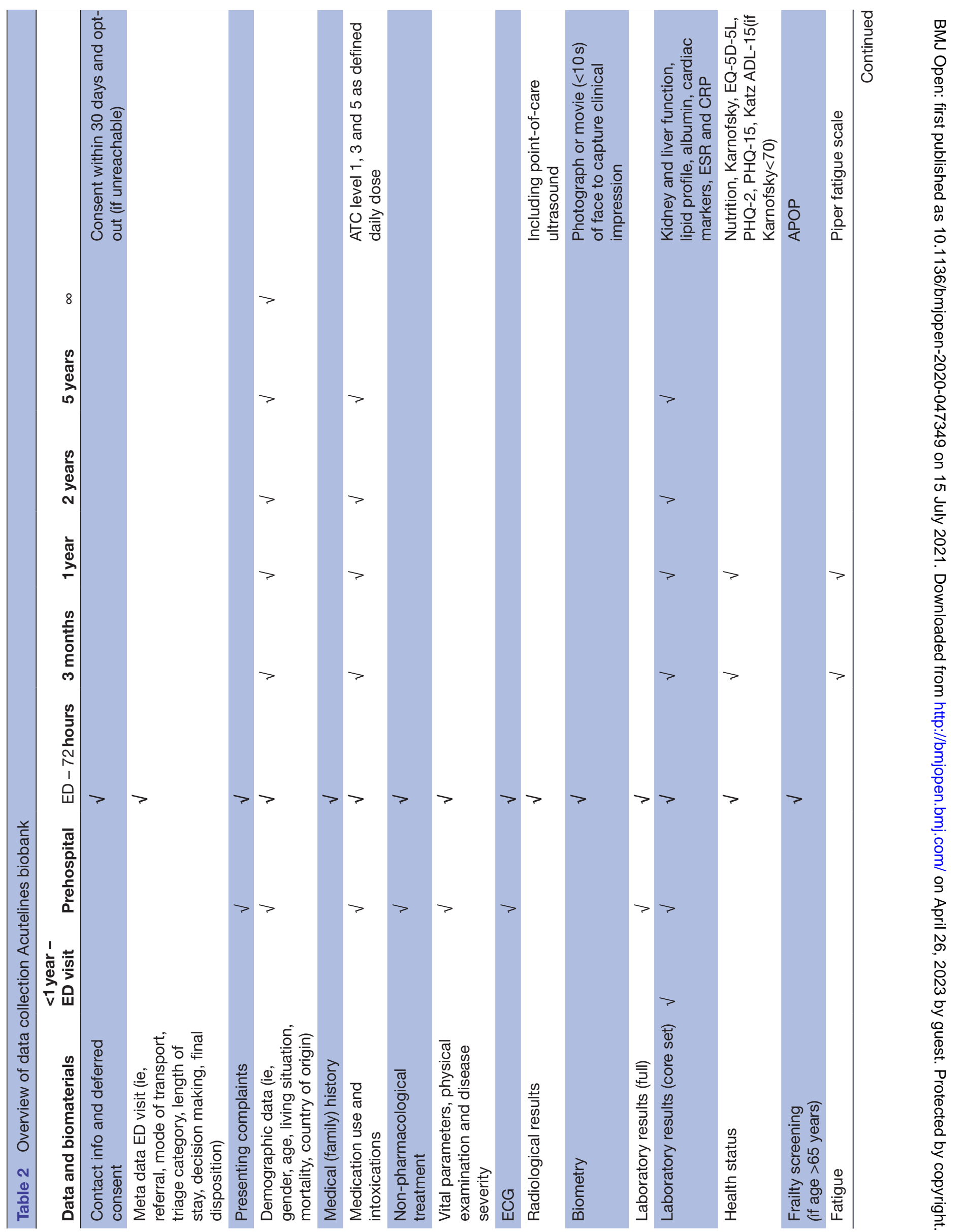


clinical impression and develop a computer-driven clinical impression using AI, we will ask participants for their permission to capture a photograph and short movie (10s) of the face of the participant. These photographs data will be stored on a secured server within the UMCG network, coded by an image identification (ID) and separate from other research data. The image ID can be used to look up the subject ID and in turn, using the EHR, identify the patient. This method precludes the ability to directly related image information to other personal data of the participant.

\section{Follow-up data}

At 3 months and 1 year after their ED visit patients are asked to fill in follow-up surveys about their health status and functioning, which will be send by email or regular mail. In addition, at 3 months, 1 year, 2 years and 5 years after the initial visit to the ER, laboratory results are retrieved from the hospital information system (when performed for routine patient care) and demographic data (including mortality), comorbidities, hospitalisations and medication use can be linked with data from the general practitioner, pharmacy, municipal registry, Dutch Statistics' Office and other registries.

\section{External data sources}

To facilitate (large scale) data importation from sources outside the hospital, we aim to make use of existing connections between healthcare databases, such as the national support centre for pharmacy (in Dutch: Landelijk Schakelpunt) the drug interaction database (IADB/ Lareb) and health insurance companies (Vektis), the integral cancer centre of the Netherlands, the PathologicalAnatomical National Automated Archive and the Dutch institute for healthcare research as much as possible. To obtain information about the potential date and cause-ofdeath, consent is sought to obtain data from the Municipal registry (in Dutch: basisregistratie personen) and the Dutch Statistics' office (in Dutch: Centraal Bureau Statistiek) containing up-to-date mortality data of all Dutch citizens. Linking these registries to the Acutelines database will be performed using pseudonymisation and encryption, preferably via a Trusted Third Party.

\section{FINDINGS TO DATE}

Enrolment of the first patient occurred on 1 September 2020. During the first month, 653 patients were screened for eligibility, of which 180 were approached as potential participants. In total 151 (84\%) provided consent for participation of which 89 patients fulfilled criteria for collection of biomaterials.

\section{COLLABORATION}

Patient and public involvement

Acutelines was initiated based on input provided by patients who expressed a specific interest in optimisation 
of early recognition and treatment of acute diseases, and into long-term patient centred outcomes (such as quality of life) of acute diseases. The Acutelines protocol and regulations were written in accordance with this interest and in line with templates provided by the institutional review board, wherein the public is represented.

\section{Data sharing}

Data and biomaterials of Acutelines can be used for future studies nested within the scope of the scientific aim of Acutelines, to facilitate interdisciplinary research on the aetiology and development of acute diseases. The data management plan is in line with current best practices, including the FAIR principles for optimal reusability of data (ie, Findable, Accessible, Interoperable and Reusable), and the general data protection regulations (GDPR) regulations. The data management plan covers aspects of data standardisation, harmonisation, security and privacy protection, Information Communication Technology (ICT) infrastructure, measures to ensure data preservation and reuse. To make the data findable for others, a description of the data is included in the Groningen Data Catalogue (https://groningendat acatalogus.nl/) and data is findable via Google Dataset Search (https://datasetsearch.research.google.com/). A detailed data dictionary is available on request via acutelines@umcg.nl.

Data and/or biomaterials can be made available after filing a data access request form (online supplemental document 2: data access request form) explaining the purpose of use, for researchers inside and outside the institute. To obtain data and/or biomaterials, researchers can submit a study proposal to the scientific board, wherein the research question is described together with type and number of biomaterials requested from the bank and a data management plan (see http://acutelines.umcg.nl/ for instructions). The board can seek epidemiological, statistical, legal or ethical advice when they evaluate the proposal based on scientific merit and solidity. If a study proposal is approved by the scientific board, subsequent medical ethical approval will have to be sought from the central review board (in Dutch: centrale toetsingscommissie) in case of non-Wet Maatschappelijk Ondersteuning (WMO)-compliant research or the medical ethical committee (in Dutch: METc) in case of WMOcompliant research. Data access will be unrestricted and non-exclusive for the purpose of the proposed study, in return for coauthorship and a financial compensation dependent on both the number of data points/biomaterial required and the funding source (academic request vs industry). When data and/or biomaterials will be sent to external parties for processing or analysis, contracts and a Material and Data Transfer Agreement will have to be drawn up and signed in coordination with the UMCG Contract Research Office. Thereafter, data and/ or biomaterials can be transferred anonymised or coded to another institute. Biometry data in the form of a photograph and small movie (10s) cannot be shared with third parties. On data extraction subjects are labelled with a project-specific identifier to prevent the possibility of cross linking large amounts of data using multiple data requests.

\section{Dissemination plan}

Results of studies performed with Acutelines data will be presented on (inter)national conferences, published in (inter) national peer-reviewed journals, and will also be made available to a broader (lay) public and Acutelines participants through the dedicated website (http:/ /acutelines.umcg/) and via social media (twitter: @Acutelines; LinkedIn: https://www.linkedin.com/company/acutelines). To facilitate outreach to general public and healthcare professionals, we request every researcher using data and/or biomaterials to submit a lay person summary for social media and the website, when publishing a scientific paper.

\section{FURTHER DETAILS}

In general, biobanks in the Netherlands do not fall within the scope of the Dutch Medical Research Involving Human Subjects Act (in Dutch: Wet medisch-wetenschappelijk onderzoek met mensen, WMO). Any future amendments to the bank protocol that may potentially impact patient safety, data collection or data analysis will need to be approved by the Central Review Board of the University Medical Center Groningen.

The biobank is compliant with the UMCG guidance for data-biobanking (available on request, in Dutch only), the Dutch Medical Treatment Agreement Act (in Dutch: Wet op de geneeskundige behandelingsovereenkomst) and with GDPR: a minimal amount of data from the specified population will be acquired and stored coded with a pseudonym (data minimisation principle), and data will only be made available on an individual level if required to answer the research question; in other cases aggregated data will be made available. The keylist that can be used to identify subjects and obtain personal data (eg, name, address and other contact information, date of birth) can only be used by researchers employed by Acutelines who specifically need this information to fulfil their function. By exception and as allowed by the Data Protection Officer of the UMCG email address, country of origin and biometry data are stored in the research database. Email address is stored in a specific field labelled 'identifier' to prevent it from being exported with data, while allowing us to send digital follow-up surveys. Country of the patient and his/her parents will be collected, since genetic background can be a risk factor and of influence on the presentation and course of certain diseases, which we explain to the patient when collecting these optional data.

Consent procedure: deferred consent (by proxy) and opt-out All potentially eligible patients arriving in the ED are requested to provide verbal consent first to collect data, 
images and biomaterials that cannot be collected at a later moment. If the patient has no capacity to consent at that moment, oral consent by a proxy will be sought. Written consent of the candidate-participant is subsequently obtained according to the principle of deferred consent: the patient is given a maximum of 30 days to read the information provided about the biobank, to ask questions (to the scientific board or to an independent expert), and to reflect on the decision to participate. If the candidate-participant is not able to give permission to participate, we will aim to obtain written permission from their legal representative according to the principle of deferred consent by proxy. In the exceptional situation that potential candidate-participants and their legal representatives cannot be reached, data/biomaterials may be stored for future research according to an optout procedure whereby the UMCG research objection registry will be checked.

Consent is asked explicitly for collection and storage of data and biomaterials for research purposes, for obtaining data from other healthcare providers involved in patient care (general practitioner, EMS, pharmacist), for contacting the patient for follow-up purposes, and finally for making the (pseudoanonymised) data available to researchers allowing them to collaborate with commercial parties. Patients are informed explicitly that their genetic code may be read from the biomaterials they provided. Additional (optional) consent is sought for automated acquisition of data from healthcare registries, including the request to access communication data and identifying data. We request permission to contact the participant again in the future (eg, for additional informed consent or data collection). Once patients decide to withdraw a previously given consent (possible at any moment), they will no longer be approached for follow-up, and they can in addition request to delete and destroy all collected data and biomaterials. Finally, patients are asked whether they would like to be informed about incidental findings that might arise.

\section{Strengths and limitations}

Our data and biobank stands out compared with other banks in several aspects. First, as mentioned, we have a dedicated research team to screen and include patients and to perform data entry, quality control and quality assurance. Further, inclusion of acutely (very) sick patients who do not have capacity to consent to participate is facilitated by a deferred consent procedure (when applicable by proxy) as well as an opt-out procedure. This allows the collection of data and biomaterials at the earliest moment in time on presentation to the ED. Our digital infrastructure enables automatically capturing of all bed-side monitor data (ie, electrophysiological waveforms, vital parameters) from every patient in the ED, as well as a secure connection with other sources if needed for specific research questions, such as the EHRs of the hospital, ambulance and general practitioner, municipal registration, health insurance companies and pharmacy. This not only facilitates data collection, but also improves data reliability. A limitation of our data and biobank is the potential inefficiency of our screening and inclusion process: all patients are screened and a lot of patients are included based on broad criteria that are present on ED presentation (eg, transport mode to hospital and urgency), while post hoc information (eg, diagnosis on hospital discharge) will be used to identify specific subjects for subsequent studies. This leaves a significant amount of data and biomaterials unused. Furthermore, clinical research in acute medicine warrants the presence of researchers in the ED waiting for patients to arrive at some moments, while at other moments, there is a risk of missing inclusions due to ED overcrowding.

\section{Twitter Ewoud ter Avest @ewoudterAvest}

Acknowledgements The authors wish to sincerely thank the first Acutelines research team, consisting of Hannah J. Aerdts, Cato J. Braakhuis, Casper J. de Wit, Yanick E. Drouven, Maaike E. Enting, Rick L. Huizinga, Wyke Kroesen, Pim F. Maas, Stijn C. Meijssen, Lise Roord, Jelle J.H. Rozendaal, Elma E. Sluiter, Sophie A. Teesink, Coen L.E. Visser, Paulien F. Engelbert, Lotte R. Hazeleger, Daniel Rossi, Esther Schwarz, Lara E.E.C. Zonneveld and Samarja Haakmeester, Tessa van Lohuizen for building and participating in the biobank.

Contributors HRB conceived the idea of the biobank, and drafted the first version of the biobank protocol, which was critically reviewed and edited by EtA, BCVM and JCtM. Data management and design and development of technical infrastructure is performed by RJvW and ST. Data acquisition and verification on a day-to-day basis is performed by STH, TTH, LEvH, FEvB and FSvdV. HRB, EtA and JCtM are steering committee members of the biobank, and responsible for design, performance surveillance, and evaluation of external requests for data use. BCvM is the biobank director. EtA drafted the manuscript of this paper describing the Acutelines Data and Biobank, which was critically reviewed by all authors before submission.

Funding The authors have not declared a specific grant for this research from any funding agency in the public, commercial or not-for-profit sectors.

Competing interests None declared.

Patient consent for publication Not required.

Ethics approval The Medical Ethics Board (in Dutch: Medisch-Ethische Toetsingscommissie, METc) and the Central Review Board (in Dutch: Centrale Toetsingscommissie, CTc) of the University Medical Center Groningen have evaluated and approved the protocol of Acutelines (2019/589), which has also been ISO certified (9001:2008 Healthcare).

Provenance and peer review Not commissioned; externally peer reviewed.

Data availability statement Data sharing not applicable as no datasets generated and/or analysed for this study. This study manuscript describes the infrastructure of the Acute Lines Biobank and as such does not contain (patient) data itself. The procedures used by the bank to release data for future studies are described extensively in the manuscript.

Supplemental material This content has been supplied by the author(s). It has not been vetted by BMJ Publishing Group Limited (BMJ) and may not have been peer-reviewed. Any opinions or recommendations discussed are solely those of the author(s) and are not endorsed by BMJ. BMJ disclaims all liability and responsibility arising from any reliance placed on the content. Where the content includes any translated material, BMJ does not warrant the accuracy and reliability of the translations (including but not limited to local regulations, clinical guidelines, terminology, drug names and drug dosages), and is not responsible for any error and/or omissions arising from translation and adaptation or otherwise.

Open access This is an open access article distributed in accordance with the Creative Commons Attribution Non Commercial (CC BY-NC 4.0) license, which permits others to distribute, remix, adapt, build upon this work non-commercially, and license their derivative works on different terms, provided the original work is properly cited, appropriate credit is given, any changes made indicated, and the use is non-commercial. See: http://creativecommons.org/licenses/by-nc/4.0/. 


\section{ORCID iDs}

Ewoud ter Avest http://orcid.org/0000-0002-1462-6130

Raymond J van Wijk http://orcid.org/0000-0002-2106-7699

Jan Cornelis ter Maaten http://orcid.org/0000-0002-0353-4011

\section{REFERENCES}

1 Densen P. Challenges and opportunities facing medical education. Trans Am Clin Climatol Assoc 2011;122:48-58.

2 Smith J, Keating L, Flowerdew L, et al. An emergency medicine research priority setting partnership to establish the top 10 research priorities in emergency medicine. Emerg Med J 2017;34:454-6.

3 van de Glind I, Berben S, Zeegers F, et al. A national research agenda for pre-hospital emergency medical services in the Netherlands: a Delphi-study. Scand J Trauma Resusc Emerg Med 2016;24:2.

4 NIV kennisagenda. Nederlandse internisten vereniging. Available: https://www.demedischspecialist.nl/sites/default/files/KennisagendaNIV.pdf [Accessed 22 Oct 2020].

5 Sole ML, Middleton A, Deaton L, et al. Enrollment challenges in critical care nursing research. Am J Crit Care 2017;26:395-400.

6 Dahlberg J, Eriksen C, Robertsen A, et al. Barriers and challenges in the process of including critically ill patients in clinical studies. Scand $J$ Trauma Resusc Emerg Med 2020;28:51.

7 Garde A, O'Hearn K, Nicholls S, et al. Reporting of consent rates in critical care studies: room for improvement. J Clin Epidemiol 2016;74:51-6.

8 Appelbaum PS. Clinical practice. Assessment of patients competence to consent to treatment. N Engl J Med 2007;357:1834-40.

9 Morley C, Unwin M, Peterson GM, et al. Emergency department crowding: a systematic review of causes, consequences and solutions. PLoS One 2018;13:e0203316.

10 Peloquin D, DiMaio M, Bierer B, et al. Disruptive and avoidable: GDPR challenges to secondary research uses of data. Eur J Hum Genet 2020;28:697-705.

11 Coopersmith CM, Wunsch H, Fink MP, et al. A comparison of critical care research funding and the financial burden of critical illness in the United States. Crit Care Med 2012;40:1072-9.

12 Jalalzadeh H, Indrakusuma R, Blankensteijn JD, et al. Design and protocol of a comprehensive multicentre Biobank for abdominal aortic aneurysms. BMJ Open 2019;9:e028858.

13 Clements CM, Anderson JR, Uhl J, et al. Biobanking in the emergency department: implementation of the Mayo clinic emergency department sepsis Biorepository. Crit Care 2014;18:P41.
14 Eitel DR, Travers DA, Rosenau AM, et al. The emergency severity index triage algorithm version 2 is reliable and valid. Acad Emerg Med 2003;10:1070-80.

15 Quinten VM, van Meurs M, Wolffensperger AE, et al. Sepsis patients in the emergency department: stratification using the clinical impression score, predisposition, infection, response and organ dysfunction score or quick sequential organ failure assessment score? Eur J Emerg Med 2018;25:328-34.

16 Singer M, Deutschman CS, Seymour CW, et al. The third International consensus definitions for sepsis and septic shock (Sepsis-3). JAMA 2016;315:801-10.

17 Perez Valdivieso JR, Bes-Rastrollo M, Monedero P, et al. Karnofsky performance score in acute renal failure as a predictor of short-term survival. Nephrology 2007;12:533-8.

18 Rabito El, Marcadenti A, da Silva Fink J, et al. Nutritional risk screening 2002, short nutritional assessment questionnaire, malnutrition screening tool, and malnutrition universal screening tool are good predictors of nutrition risk in an emergency service. Nutr Clin Pract 2017;32:526-32.

19 Laan W, Zuithoff NPA, Drubbel I, et al. Validity and reliability of the Katz-15 scale to measure unfavorable health outcomes in community-dwelling older people. J Nutr Health Aging 2014;18:848-54.

20 Lakkis NA, Mahmassani DM. Screening instruments for depression in primary care: a Concise review for clinicians. Postgrad Med 2015;127:99-106.

21 Reeve BB, Stover AM, Alfano CM, et al. The Piper fatigue Scale-12 (PFS-12): psychometric findings and item reduction in a cohort of breast cancer survivors. Breast Cancer Res Treat 2012;136:9-20.

22 Gierk B, Kohlmann S, Toussaint A, et al. Assessing somatic symptom burden: a psychometric comparison of the patient health questionnaire-15 (PHQ-15) and the somatic symptom scale-8 (SSS8). J Psychosom Res 2015;78:352-5.

23 de Gelder J, Lucke JA, Blomaard LC, et al. Optimization of the apop screener to predict functional decline or mortality in older emergency department patients: cross-validation in four prospective cohorts. Exp Gerontol 2018;110:253-9.

24 Harris PA, Taylor R, Thielke R, et al. Research electronic data capture (REDCap)--a metadata-driven methodology and workflow process for providing translational research informatics support. J Biomed Inform 2009;42:377-81.

25 Harris PA, Taylor R, Minor BL, et al. The REDCap Consortium: building an international community of software platform partners. $J$ Biomed Inform 2019;95:103208. 